\title{
FINITENESS CRITERIA \\ IN GORENSTEIN HOMOLOGICAL ALGEBRA
}

\author{
IOANNIS EMMANOUIL AND OLYMPIA TALELLI
}

\begin{abstract}
In this paper, we examine the class of modules of finite Gorenstein projective dimension and study approximations of modules in that class by modules which are either Gorenstein projective or else have finite projective dimension. We also examine the relevance of complete cohomology in the study of modules of finite Gorenstein projective dimension and obtain, over group rings, such a finiteness criterion that involves only complete cohomology.
\end{abstract}

\section{Contents}

0. Introduction

1. Modules of finite Gorenstein projective dimension

2. Stabilizing with respect to modules of finite projective dimension

3. Stabilizing with respect to Gorenstein projective modules

4. Finiteness of Gorenstein projective dimension and complete cohomology

5. Modules of finite Gorenstein injective dimension

6 . The special case of group rings

\section{INTRODUCTION}

Auslander and Bridger defined in [1, 2, the G-dimension G-dim ${ }_{R} M$ of any finitely generated module $M$ over a commutative Noetherian local ring $R$ and showed that $R$ is Gorenstein if and only if the G-dimension of its residue field is finite. The G-dimension generalizes the projective dimension, in the sense that if $M$ is a finitely generated module of finite projective dimension, then G- $\operatorname{dim}_{R} M=\operatorname{pd}_{R} M$. This definition was extended to all modules over any ring $R$ by Enochs and Jenda in [14: A module is said to be Gorenstein projective if it is a syzygy of a doubly infinite acyclic complex of projective modules

$$
\cdots \longrightarrow P_{n+1} \longrightarrow P_{n} \longrightarrow P_{n-1} \longrightarrow \cdots,
$$

which remains acyclic when applying the functor $\operatorname{Hom}_{R}(-, P)$ for any projective module $P$. Then, modules of finite Gorenstein projective dimension are defined in the standard way, by using resolutions by Gorenstein projective modules. As shown

\footnotetext{
Received by the editors March 6, 2012 and, in revised form, October 14, 2012.

2010 Mathematics Subject Classification. Primary 16E10, 18G20; Secondary 20J05.

This research was supported by a GSRT/Greece excellence grant, cofunded by the ESF/EU and National Resources. 
in [10, Theorem 4.2.6], the Gorenstein projective dimension of a finitely generated module over a commutative Noetherian local ring agrees with its G-dimension. The relative cohomology theory obtained by resolving the first argument of the Hom functor by Gorenstein projective modules has been studied in detail in 4] for modules of finite Gorenstein projective dimension; for such modules, the relative theory is closely related to both the ordinary and the complete cohomology.

The concept of a maximal Cohen-Macaulay approximation, which was introduced and studied by Auslander and Buchweitz in [3] for finitely generated modules over a commutative Gorenstein local ring, has been generalized by Holm who showed in [18, Theorem 2.10] that if $R$ is any ring and $M$ is an $R$-module of finite Gorenstein projective dimension, then there exists a short exact sequence of $R$-modules

$$
0 \longrightarrow K \longrightarrow G \longrightarrow M \longrightarrow 0,
$$

where $G$ is Gorenstein projective and $\operatorname{pd}_{R} K<\infty$. Such an exact sequence satisfies a weak form of uniqueness, as in the classical Schanuel's lemma. We shall describe in section 2 the precise sense in which $G$ may be regarded as an approximation of $M$ by a Gorenstein projective module: We consider the stabilization $\mathfrak{F P}-R$-Mod of the category of $R$-modules with respect to the class of modules of finite projective dimension and denote by $\mathfrak{F P}-\mathrm{FGP}(R)$ (resp. by $\mathfrak{F P}-\mathrm{GP}(R)$ ) the full subcategory of $\mathfrak{F P}-R$-Mod consisting of the modules of finite Gorenstein projective dimension (resp. of the Gorenstein projective modules). Then, the assignment $M \mapsto G$ extends to a functor $\mu: \mathfrak{F P}-\mathrm{FGP}(R) \longrightarrow \mathfrak{F P}-\mathrm{GP}(R)$, which is right adjoint to the inclusion functor $\mathfrak{F P}-\mathrm{GP}(R) \hookrightarrow \mathfrak{F P}-\mathrm{FGP}(R)$. Vanishing of the counit of this adjunction characterizes the modules of finite projective dimension among the modules of finite Gorenstein projective dimension.

As shown in [11, Lemma 2.17], one may use the infinite-loop-space-like structure of the class of Gorenstein projective modules in order to dualize (1) and obtain for any module $M$ of finite Gorenstein projective dimension an exact sequence of $R$-modules

$$
0 \longrightarrow M \longrightarrow A \longrightarrow G^{\prime} \longrightarrow 0,
$$

where $G^{\prime}$ is Gorenstein projective and $\operatorname{pd}_{R} A<\infty$. As above, such an exact sequence satisfies a weak form of uniqueness (à la Schanuel). We shall explain in section 3 the precise sense in which $A$ may be regarded as an approximation of $M$ by a module of finite projective dimension: We consider the stabilization $\mathfrak{G P}-R$-Mod of the category of $R$-modules with respect to the class of Gorenstein projective modules and denote by $\mathfrak{G P}-\mathrm{FGP}(R)$ (resp. by $\mathfrak{G P}-\mathrm{FP}(R)$ ) the full subcategory of $\mathfrak{G P}-R$-Mod consisting of the modules of finite Gorenstein projective dimension (resp. of finite projective dimension). Then, the assignment $M \mapsto A$ extends to a functor $\nu: \mathfrak{G P}-\mathrm{FGP}(R) \longrightarrow \mathfrak{G P}-\mathrm{FP}(R)$, which is left adjoint to the inclusion functor $\mathfrak{G P}-\mathrm{FP}(R) \hookrightarrow \mathfrak{G P}-\mathrm{FGP}(R)$. Vanishing of the unit of this adjunction characterizes the Gorenstein projective modules among the modules of finite Gorenstein projective dimension.

The two reformulations of the finiteness of the Gorenstein projective dimension of a module $M$, which are described by the existence of short exact sequences as in (1) and (2) above, are somehow analogous to the interpretations of an element $\alpha \in \operatorname{Ext}_{R}^{n}(X, Y)$ (where $X, Y$ are two modules and $n$ is a positive integer) in classical homological algebra. On one hand, $\alpha$ may be described by using a projective 
resolution $P_{*} \longrightarrow X \longrightarrow 0$ as the cohomology class of a cocycle $f: \Omega^{n} X \longrightarrow Y$. This interpretation is analogous to the derivation of the exact sequence (1) out of the assumption that the module $M$ has finite Gorenstein projective dimension; cf. the proof of [18, Theorem 2.10] and [4, Construction 5.10]. Alternatively, we may use Yoneda's approach and describe $\alpha$ as the equivalence class of an $n$-fold extension

$$
0 \longrightarrow Y \longrightarrow Z \longrightarrow P_{n-2} \longrightarrow \cdots \longrightarrow P_{0} \longrightarrow X \longrightarrow 0
$$

where the modules $P_{0}, \ldots, P_{n-2}$ are projective. One may construct such an extension by starting from a projective resolution $P_{*} \longrightarrow X \longrightarrow 0$ and a cocycle $f: \Omega^{n} X \longrightarrow Y$ that represents $\alpha$ and then defining $Z$ to be the pushout of $f$ and the inclusion $\Omega^{n} X \hookrightarrow P_{n-1}$. This approach is analogous to the derivation of the exact sequence (2) out of the exact sequence (1), which is presented in the proof of [11, Lemma 2.17] (and reproduced in the proof of Proposition 1.2 below).

The conditions characterizing the finiteness of the Gorenstein projective dimension of a module $M$ that are described by the existence of short exact sequences as in (1) and (2) above may be relaxed by removing the assumption that the modules $K$ and $A$ therein have finite projective dimension and assuming instead that certain elements of complete cohomology groups vanish. Using the complete cohomology functors $\widehat{\operatorname{Ext}}_{R}^{*}(-,-)$, which were defined by Benson, Carlson, Mislin and Vogel in [7], [17] and [21, we prove that a module $M$ has finite Gorenstein projective dimension if and only if one of the following two conditions is satisfied:

(i) There exists a short exact sequence of $R$-modules $0 \longrightarrow K \longrightarrow G \longrightarrow$ $M \longrightarrow 0$, where $G$ is Gorenstein projective, such that the image of the element $\xi \in \operatorname{Ext}_{R}^{1}(M, K)$ that classifies the extension vanishes in the group $\widehat{\operatorname{Ext}}_{R}^{1}(M, K)$.

(ii) There exists a short exact sequence of $R$-modules $0 \longrightarrow M \stackrel{\iota}{\longrightarrow} A \longrightarrow G^{\prime} \longrightarrow$ 0 , where $G^{\prime}$ is Gorenstein projective, such that the image of $\iota \in \operatorname{Hom}_{R}(M, A)$ vanishes in the group $\widehat{\operatorname{Ext}}_{R}^{0}(M, A)$.

It is interesting to note that conditions (i) and (ii) above involve no a priori assumption about the finiteness of the projective dimension of the modules $K$ and $A$ therein.

We now consider the special case where $R$ is the integral group ring $\mathbb{Z} G$ of a group $G$ and examine the Gorenstein cohomological dimension $\operatorname{Gcd}_{\mathbb{Z}} G$ of $G$ over $\mathbb{Z}$, which is defined to be the Gorenstein projective dimension of the trivial $\mathbb{Z} G$-module $\mathbb{Z}$. It follows from the discussion above that $\operatorname{Gcd}_{\mathbb{Z}} G<\infty$ if and only if there exists a short exact sequence of $\mathbb{Z} G$-modules

$$
0 \longrightarrow \mathbb{Z} \longrightarrow A \longrightarrow \bar{A} \longrightarrow 0,
$$

where $\operatorname{pd}_{\mathbb{Z} G} A<\infty$ and $\bar{A}$ is Gorenstein projective. A stronger version of that characterization was obtained in [6, Theorem 2.7], where the finiteness of the Gorenstein cohomological dimension of $G$ over $\mathbb{Z}$ was shown to be equivalent to the existence of a $\mathbb{Z}$-split monomorphism of $\mathbb{Z} G$-modules $\mathbb{Z} \longrightarrow A$, where $A$ is $\mathbb{Z}$-free and $\operatorname{pd}_{\mathbb{Z} G} A<\infty$. This result shows that the finiteness of the Gorenstein cohomological dimension of $G$ over $\mathbb{Z}$ may be described solely in terms of ordinary group cohomology. Using in an essential way the Hopficity of the group ring $\mathbb{Z} G$, we shall obtain in section 6 an even stronger version of the latter characterization. We prove that the finiteness of $\operatorname{Gcd}_{\mathbb{Z}} G$ is equivalent to the existence of a $\mathbb{Z}$-split monomorphism of $\mathbb{Z} G$-modules $\iota: \mathbb{Z} \longrightarrow A$, where $A$ is $\mathbb{Z}$-free, such that the image 
of $\iota \in \operatorname{Hom}_{\mathbb{Z} G}(\mathbb{Z}, A)=H^{0}(G, A)$ vanishes in the group $\widehat{H}^{0}(G, A)$. Hence, the finiteness of the Gorenstein cohomological dimension of $G$ over $\mathbb{Z}$ may also be described solely in terms of complete cohomology. Note that this result is stronger than the characterization of finiteness described in condition (ii) above, in the case of the trivial $\mathbb{Z} G$-module $\mathbb{Z}$, since there is no additional assumption about the module $\bar{A}=$ coker $\iota$ being Gorenstein projective.

The Gorenstein cohomological dimension $\operatorname{Gcd}_{\mathbb{Z}} G$ is a generalization of the ordinary cohomological dimension $\operatorname{cd}_{\mathbb{Z}} G$ of $G$ over $\mathbb{Z}$ : If $\operatorname{cd}_{\mathbb{Z}} G$ is finite, then we have an equality $\operatorname{Gcd}_{\mathbb{Z}} G=\operatorname{cd}_{\mathbb{Z}} G$. On the other hand, it is easily seen that the vanishing of $\operatorname{Gcd}_{\mathbb{Z}} G$ characterizes the finite groups, i.e. $\operatorname{Gcd}_{\mathbb{Z}} G=0$ if and only if the group $G$ is finite. We note that the cohomological dimension $\operatorname{cd}_{\mathbb{Z}} G$ of any non-trivial finite group $G$ is infinite. C.T.C. Wall has proved in $\left[24\right.$ that $\operatorname{cd}_{\mathbb{Z}} G<\infty$ if and only if $G$ acts freely and cellularly on a contractible finite dimensional CW-complex. We expect that the finiteness of the Gorenstein cohomological dimension $\operatorname{Gcd}_{\mathbb{Z}} G$ of $G$ over $\mathbb{Z}$ should play a central role in any attempt to generalize Wall's theorem, in a way that includes actions with finite stabilizers. Indeed, if $G$ acts cellularly with finite stabilizers on a contractible finite dimensional CW-complex $X$, then the cellular chain complex of $X$ is a Gorenstein projective resolution of $G$, i.e. a resolution of the trivial $\mathbb{Z} G$-module $\mathbb{Z}$ by Gorenstein projective modules.

Notation and terminology. Unless otherwise specified, all modules considered in this paper will be left modules over a fixed $\operatorname{ring} R$. For any module $M$ we shall denote by $\Omega M$ the kernel of a surjective $R$-linear map $P \longrightarrow M$, where $P$ is a projective module. Even though $\Omega M$ is not uniquely determined by $M$, Schanuel's lemma implies uniqueness up to addition of a projective module. We also consider the iterates $\Omega^{n} M, n \geq 1$, which are defined inductively by letting $\Omega^{1} M=\Omega M$ and $\Omega^{n} M=\Omega \Omega^{n-1} M$ for all $n>1$. If $P_{*} \longrightarrow M \longrightarrow 0$ is a projective resolution of $M$, then $\Omega^{n} M$ is identified with the image of the map $P_{n} \longrightarrow P_{n-1}$ for all $n \geq 1$. A doubly infinite acyclic complex of projective modules is called totally acyclic if the complex remains exact after applying the functor $\operatorname{Hom}_{R}(-, P)$ for any projective module $P$. In this way, the Gorenstein projective modules are precisely the syzygies of the totally acyclic complexes of projective modules.

\section{Modules of finite Gorenstein projective Dimension}

In this preliminary section, we shall review a few equivalent formulations of the finiteness of the Gorenstein projective dimension of a module and record some consequences concerning the relation between modules of finite Gorenstein projective dimension, modules of finite projective dimension and Gorenstein projective modules.

Lemma 1.1. A module $M$ is Gorenstein projective if and only if there exists a short exact sequence of $R$-modules $0 \longrightarrow M \longrightarrow P \longrightarrow G \longrightarrow 0$, where $P$ is projective and $G$ is Gorenstein projective.

Proof. If $M$ is Gorenstein projective, then we may obtain a short exact sequence as in the statement by considering a totally acyclic complex of projective modules that has $M$ as a syzygy. Conversely, the existence of such a short exact sequence implies that the module $M$ is Gorenstein projective, in view of [18, Theorem 2.5].

The next result (more precisely, the equivalence between assertions (i) and (iii) therein) is a higher dimensional analogue of the above lemma. 
Proposition 1.2. The following conditions are equivalent for a module $M$ and $a$ non-negative integer $n$ :

(i) $\operatorname{Gpd}_{R} M \leq n$,

(ii) there exists a short exact sequence of $R$-modules $0 \longrightarrow K \longrightarrow G \longrightarrow M \longrightarrow$ 0 , where $G$ is Gorenstein projective and $p d_{R} K \leq n-1$ (if $n=0$, this is understood to mean $K=0$ ), and

(iii) there exists a short exact sequence of $R$-modules $0 \longrightarrow M \longrightarrow A \longrightarrow G^{\prime} \longrightarrow$ 0 , where $G^{\prime}$ is Gorenstein projective and $p d_{R} A \leq n$.

Proof. The equivalence between (i) and (ii) has been shown in [18, Theorem 2.10]. The implication (ii) $\rightarrow$ (iii) has been proved in [11, Lemma 2.17], as follows: Assume that there exists a short exact sequence of $R$-modules as in (ii). Then, Lemma 1.1 implies that $G$ fits into a short exact sequence of $R$-modules

$$
0 \longrightarrow G \longrightarrow P \longrightarrow G^{\prime} \longrightarrow 0
$$

where $P$ is projective and $G^{\prime}$ is Gorenstein projective. We consider the pushout of the diagram

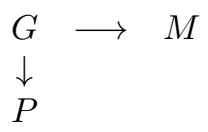

which fits into a commutative diagram with exact rows and columns:

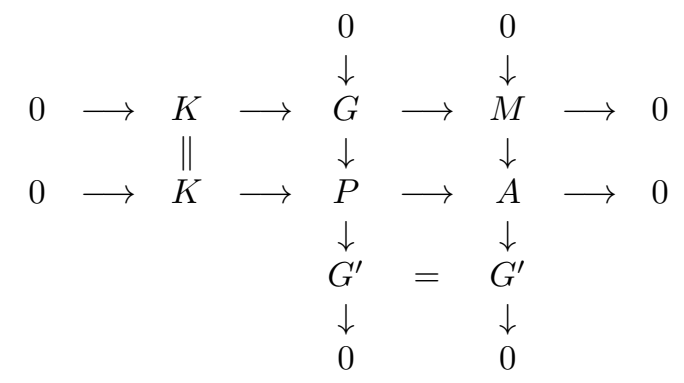

Since $\operatorname{pd}_{R} K \leq n-1$, the horizontal exact sequence in the middle of the diagram shows that $\operatorname{pd}_{R} A \leq n$. Hence, the rightmost vertical exact sequence has all of the desired properties.

(iii) $\rightarrow$ (ii): Assume that there exists a short exact sequence of $R$-modules as in (iii). Since $\operatorname{pd}_{R} A \leq n$, there exists a short exact sequence of $R$-modules

$$
0 \longrightarrow K \longrightarrow P \longrightarrow A \longrightarrow 0
$$

where $P$ is projective and $\operatorname{pd}_{R} K \leq n-1$. We now consider the pullback of the diagram

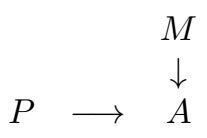


which fits into a commutative diagram with exact rows and columns:

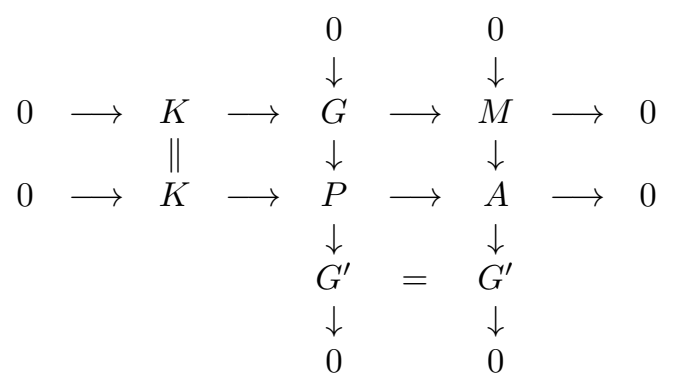

Since $G^{\prime}$ is Gorenstein projective, we may apply Lemma 1.1 to the vertical exact sequence in the middle of the diagram in order to conclude that $G$ is Gorenstein projective as well. Hence, the top horizontal exact sequence has all of the desired properties.

Remarks 1.3. (a) Let $M$ be a module of finite Gorenstein projective dimension. Then, the two constructions described in the proof of the equivalence between conditions (ii) and (iii) in Proposition 1.2 above are inverse to each other, provided that we make the obvious choices in the second step in each direction. (What we mean by this is that having performed the construction described in the proof of the implication (ii) $\rightarrow$ (iii) in the short exact sequence $0 \longrightarrow K \longrightarrow G \longrightarrow M \longrightarrow 0$, there is an obvious choice for a description of $A$ as a quotient of the form $P / K$. Similarly, having performed the construction described in the proof of the implication (iii) $\rightarrow$ (ii) in the short exact sequence $0 \longrightarrow M \longrightarrow A \longrightarrow G^{\prime} \longrightarrow 0$, there is an obvious choice for a description of $G$ as the kernel of a surjective map $P \longrightarrow G^{\prime}$.) This assertion follows from two elementary observations:

(a1) If the commutative square

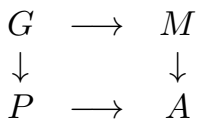

is a pushout diagram and the morphism $G \longrightarrow P$ is injective, then the square is also a pullback diagram.

(a2) If the commutative square

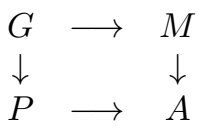

is a pullback diagram and the morphism $P \longrightarrow A$ is surjective, then the square is also a pushout diagram.

(b) We may restate the equivalence between conditions (ii) and (iii) in Proposition 1.2 as follows: Any short exact sequence of $R$-modules $0 \longrightarrow M \longrightarrow A \longrightarrow$ $G^{\prime} \longrightarrow 0$ as in Proposition 1.2(iii) induces a short exact sequence $0 \longrightarrow \Omega A \longrightarrow$ $\Omega G^{\prime} \longrightarrow M \longrightarrow 0$, which is of the form required in Proposition 1.2(ii), since the operation $\Omega$ maps modules of finite projective dimension (resp. Gorenstein projective modules) onto modules of finite projective dimension (resp. onto Gorenstein projective modules). Conversely, for any short exact sequence of $R$-modules $0 \longrightarrow K \longrightarrow G \longrightarrow M \longrightarrow 0$ as in Proposition 1.2(ii), we may express $G$ as $\Omega G^{\prime}$ for some Gorenstein projective module $G^{\prime}$. Then, there is an induced description 
of $K$ as $\Omega A$ for some module $A$, which has necessarily finite projective dimension. Hence, the given exact sequence is of the form $0 \longrightarrow \Omega A \longrightarrow \Omega G^{\prime} \longrightarrow M \longrightarrow 0$ and thus induces a short exact sequence $0 \longrightarrow M \longrightarrow A \longrightarrow G^{\prime} \longrightarrow 0$, which is of the form required in Proposition 1.2(iii).

Even though the exact sequences described in Proposition 1.2(ii) and (iii) are by no means unique, they do satisfy a weak form of uniqueness (à la Schanuel), as shown below.

Lemma 1.4. Let $M$ be a module of finite Gorenstein projective dimension.

(i) Assume that $0 \longrightarrow K \longrightarrow G \longrightarrow M \longrightarrow 0$ and $0 \longrightarrow \bar{K} \longrightarrow \bar{G} \longrightarrow M \longrightarrow 0$ are two short exact sequences of $R$-modules with $K, \bar{K}$ of finite projective dimension and $G, \bar{G}$ Gorenstein projective. Then, there is an isomorphism $G \oplus \bar{K} \simeq \bar{G} \oplus K$.

(ii) Assume that $0 \longrightarrow M \longrightarrow A \longrightarrow G^{\prime} \longrightarrow 0$ and $0 \longrightarrow M \longrightarrow \bar{A} \longrightarrow \overline{G^{\prime}} \longrightarrow 0$ are two short exact sequences of $R$-modules with $A, \bar{A}$ of finite projective dimension and $G^{\prime}, \overline{G^{\prime}}$ Gorenstein projective. Then, there is an isomorphism $G^{\prime} \oplus \bar{A} \simeq \overline{G^{\prime}} \oplus A$.

Proof. (i) Since $G$ is Gorenstein projective and $\operatorname{pd}_{R} \bar{K}<\infty$, the group $\operatorname{Ext}_{R}^{1}(G, \bar{K})$ is trivial, and hence there exists an $R$-linear map $\lambda: G \longrightarrow \bar{G}$ making the right square in the following diagram with exact rows commutative:

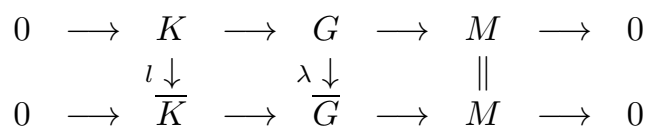

Then, there exists a unique $R$-linear map $l: K \longrightarrow \bar{K}$, which makes the left square of the diagram commutative as well. We may view the pair $(l, \lambda)$ as a quasiisomorphism between the complexes $0 \longrightarrow K \longrightarrow G \longrightarrow 0$ and $0 \longrightarrow \bar{K} \longrightarrow \bar{G} \longrightarrow$ 0 and consider the corresponding mapping cone (which is an acyclic complex). We therefore obtain a short exact sequence

$$
0 \longrightarrow K \longrightarrow G \oplus \bar{K} \longrightarrow \bar{G} \longrightarrow 0 .
$$

Since $\bar{G}$ is Gorenstein projective and $\operatorname{pd}_{R} K<\infty$, the group $\operatorname{Ext}_{R}^{1}(\bar{G}, K)$ is trivial, and hence the above exact sequence splits. It follows that there exists an isomorphism $G \oplus \bar{K} \simeq \bar{G} \oplus K$, as needed.

(ii) We use an argument, which is similar to that used in the proof of (i) above: Since $G^{\prime}$ is Gorenstein projective and $\operatorname{pd}_{R} \bar{A}<\infty$, the group $\operatorname{Ext}_{R}^{1}\left(G^{\prime}, \bar{A}\right)$ is trivial, and hence there exists an $R$-linear map $\varrho: A \longrightarrow \bar{A}$ making the left square in the following diagram with exact rows commutative:

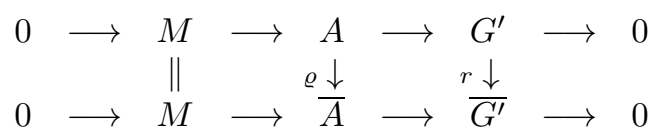

Then, there exists a unique $R$-linear map $r: G^{\prime} \longrightarrow \overline{G^{\prime}}$, which makes the right square of the diagram commutative as well. We may view the pair $(\varrho, r)$ as a quasiisomorphism between the complexes $0 \longrightarrow A \longrightarrow G^{\prime} \longrightarrow 0$ and $0 \longrightarrow \bar{A} \longrightarrow \overline{G^{\prime}} \longrightarrow$ 0 and consider the corresponding mapping cone (which is an acyclic complex). We therefore obtain a short exact sequence

$$
0 \longrightarrow A \longrightarrow G^{\prime} \oplus \bar{A} \longrightarrow \overline{G^{\prime}} \longrightarrow 0 .
$$


Since $\overline{G^{\prime}}$ is Gorenstein projective and $\operatorname{pd}_{R} A<\infty$, the group $\operatorname{Ext}_{R}^{1}\left(\overline{G^{\prime}}, A\right)$ is trivial, and hence the above exact sequence splits. It follows that there exists an isomorphism $G^{\prime} \oplus \bar{A} \simeq \overline{G^{\prime}} \oplus A$, as needed.

As an immediate consequence of Proposition 1.2, we may obtain a simple characterization of those modules of finite Gorenstein projective dimension which are Gorenstein projective (or have finite projective dimension), in terms of the functor $\operatorname{Ext}_{R}^{1}$.

Corollary 1.5. Let $M$ be a module of finite Gorenstein projective dimension. Then:

(i) $M$ is Gorenstein projective if and only if the functor $\operatorname{Ext}_{R}^{1}(M,-)$ vanishes on all modules of finite projective dimension and

(ii) $M$ has finite projective dimension if and only if the functor $\operatorname{Ext}_{R}^{1}(-, M)$ vanishes on all Gorenstein projective modules.

Proof. (i) If $M$ is Gorenstein projective, then $\operatorname{Ext}_{R}^{1}(M, A)=0$ for all modules $A$ of finite projective dimension. Conversely, assume that this condition is satisfied and consider a short exact sequence of $R$-modules

$$
0 \longrightarrow K \longrightarrow G \longrightarrow M \longrightarrow 0,
$$

where $G$ is Gorenstein projective and $\operatorname{pd}_{R} K<\infty$. Since the group $\operatorname{Ext}_{R}^{1}(M, K)$ is trivial, the above exact sequence splits. Then, $M$ is a direct summand of the Gorenstein projective module $G$ and hence is itself Gorenstein projective (cf. [18, Theorem 2.5]).

(ii) If $M$ has finite projective dimension, then $\operatorname{Ext}_{R}^{1}(G, M)=0$ for all Gorenstein projective modules $G$. Conversely, assume that this condition is satisfied and consider a short exact sequence of $R$-modules

$$
0 \longrightarrow M \longrightarrow A \longrightarrow G^{\prime} \longrightarrow 0,
$$

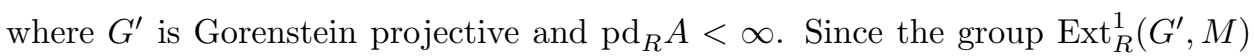
is trivial, the above exact sequence splits. Then, $M$ is a direct summand of the module $A$ and hence has finite projective dimension; in fact, $\operatorname{pd}_{R} M \leq \operatorname{pd}_{R} A$.

If $\mathfrak{X}$ is a class of modules, then the left (resp. right) Ext ${ }^{1}$-orthogonal of $\mathfrak{X}$ is the class ${ }^{\perp} \mathfrak{X}\left(\right.$ resp. $\left.\mathfrak{X}^{\perp}\right)$, consisting of those modules $Y$ for which the group $\operatorname{Ext}_{R}^{1}(Y, X)$ (resp. $\left.\operatorname{Ext}_{R}^{1}(X, Y)\right)$ is trivial for all $X \in \mathfrak{X}$. In this way, we may rephrase Corollary 1.5 by saying that ${ }^{\perp} \mathrm{FP}(R) \cap \mathrm{FGP}(R)=\mathrm{GP}(R)$ and $\mathrm{GP}(R)^{\perp} \cap \mathrm{FGP}(R)=\mathrm{FP}(R)$, where $\mathrm{FP}(R)$ (resp. $\mathrm{FGP}(R)$, resp. $\mathrm{GP}(R)$ ) is the class of all modules of finite projective dimension (resp. of all modules of finite Gorenstein projective dimension, resp. of all Gorenstein projective modules). In particular, we may state the following corollary, dealing with rings $R$ of finite left Gorenstein global dimension; these are precisely those rings $R$ over which the class FGP $(R)$ comprises of all modules.

Corollary 1.6. If $R$ is a ring of finite left Gorenstein global dimension, then ${ }^{\perp} \mathrm{FP}(R)=\mathrm{GP}(R)$ and $\mathrm{GP}(R)^{\perp}=\mathrm{FP}(R)$.

Corollary 1.7. Let $M$ be a module of finite Gorenstein projective dimension and consider a non-negative integer $n$. Then, $\operatorname{Gpd}_{R} M \leq n$ if and only if the functor $\operatorname{Ext}_{R}^{n+1}\left(M,_{-}\right)$vanishes on all modules of finite projective dimension. 
Proof. Since $M$ has finite Gorenstein projective dimension, the $n$-th syzygy module $\Omega^{n} M$ has finite Gorenstein projective dimension as well (cf. [18, Theorem 2.24]). Moreover, we have $\operatorname{Gpd}_{R} M \leq n$ if and only if $\Omega^{n} M$ is Gorenstein projective (cf. [18, Proposition 2.7]). Since the functors $\operatorname{Ext}_{R}^{1}\left(\Omega^{n} M,_{-}\right)$and $\operatorname{Ext}_{R}^{n+1}\left(M,_{-}\right)$are naturally isomorphic, the result is an immediate consequence of Corollary 1.5(i).

\section{Stabilizing With Respect to modules of Finite projective Dimension}

Let $M, N$ be two modules. Then, the set consisting of those $R$-linear maps $f: M \longrightarrow N$ that factor through a module of finite projective dimension is a subgroup of the abelian group $\operatorname{Hom}_{R}(M, N)$. (This follows since the direct sum of two modules of finite projective dimension has finite projective dimension as well.) We shall denote by $\mathfrak{F P}-\operatorname{Hom}_{R}(M, N)$ the corresponding quotient group and let $[f]=[f]_{\mathfrak{F} \mathfrak{P}}$ be the class of any $R$-linear map $f \in \operatorname{Hom}_{R}(M, N)$ therein. We note that for any three modules $M, N$ and $L$ the composition of $R$-linear maps induces a well-defined biadditive map

$$
\mathfrak{F P}^{-H_{o m}}(N, L) \times \mathfrak{F P}-\operatorname{Hom}_{R}(M, N) \longrightarrow \mathfrak{F P}-\operatorname{Hom}_{R}(M, L) .
$$

We shall denote by $\mathfrak{F P}$ - $R$-Mod the category whose objects are all modules and whose morphism sets are given by the abelian groups $\mathfrak{F P}-\operatorname{Hom}_{R}(M, N)$ (with the composition of morphisms induced by the composition of $R$-linear maps). Then, Lemma 1.4 implies that the Gorenstein projective modules $G$ and $G^{\prime}$, which are defined for any module $M$ of finite Gorenstein projective dimension in Proposition 1.2(ii) and (iii), are uniquely determined up to isomorphism as objects of the category $\mathfrak{F P}$ - $R$-Mod.

Lemma 2.1. Let $f: M \longrightarrow N$ be an R-linear map, where $M, N$ are two modules of finite Gorenstein projective dimension. We also consider two exact sequences of $R$-modules,

$$
0 \longrightarrow K \stackrel{\iota}{\longrightarrow} G \stackrel{p}{\longrightarrow} M \longrightarrow 0 \text { and } 0 \longrightarrow L \stackrel{3}{\longrightarrow} H \stackrel{q}{\longrightarrow} N \longrightarrow 0,
$$

where $K, L$ have finite projective dimension and $G, H$ are Gorenstein projective. Then:

(i) There exists an $R$-linear map $g: G \longrightarrow H$, such that $q g=f p$.

(ii) If $g, g^{\prime}: G \longrightarrow H$ are two R-linear maps with $q g=f p$ and $q g^{\prime}=f p$, then $[g]=\left[g^{\prime}\right] \in \mathfrak{F P}-H_{o m}(G, H)$.

(iii) If $[f]=[0] \in \mathfrak{F P}-H_{R}(M, N)$ and $g: G \longrightarrow H$ is an $R$-linear map such that $q g=f p$, then $[g]=[0] \in \mathfrak{F P}-\operatorname{Hom}_{R}(G, H)$.

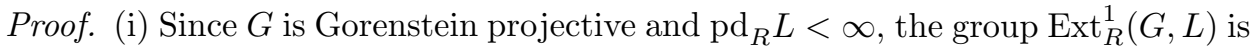
trivial, and hence the additive map $q_{*}: \operatorname{Hom}_{R}(G, H) \longrightarrow \operatorname{Hom}_{R}(G, N)$ is surjective. Therefore, there exists an $R$-linear map $g: G \longrightarrow H$ such that $f p=q_{*}(g)$, as needed.

(ii) Let $g, g^{\prime}: G \longrightarrow H$ be two $R$-linear maps with $q g=f p$ and $q g^{\prime}=f p$ :

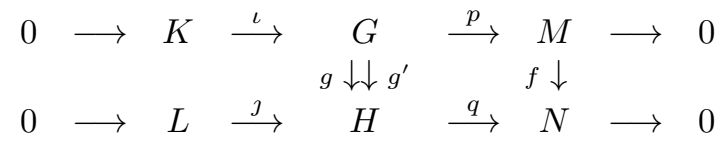

Then, $q\left(g^{\prime}-g\right)=q g^{\prime}-q g=f p-f p=0$, and hence there exists an $R$-linear map $h: G \longrightarrow L$, such that $g^{\prime}-g=\jmath h$. Since $L$ has finite projective dimension, we conclude that $[g]=\left[g^{\prime}\right] \in \mathfrak{F P}-\operatorname{Hom}_{R}(G, H)$. 
(iii) Assume that $f$ factors as the composition of two $R$-linear maps $M \stackrel{a}{\longrightarrow}$ $\Lambda \stackrel{b}{\longrightarrow} N$, where the module $\Lambda$ has finite projective dimension. Let

$$
0 \longrightarrow \Lambda^{\prime} \longrightarrow P \stackrel{\pi}{\longrightarrow} \Lambda \longrightarrow 0
$$

be a short exact sequence of $R$-modules with $P$ projective (and $\Lambda^{\prime}$ of finite projective dimension). We may construct such an exact sequence by considering the beginning of a projective resolution of $\Lambda$. Then, invoking (i) above, we may find $R$-linear maps $\alpha: G \longrightarrow P$ and $\beta: P \longrightarrow H$, such that $\pi \alpha=a p$ and $q \beta=b \pi:$

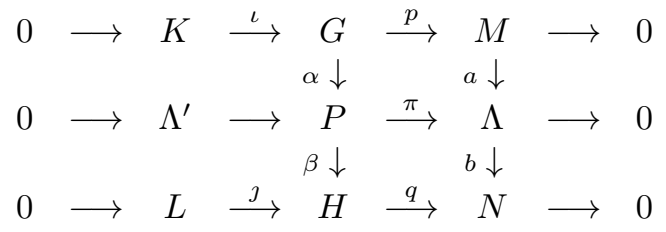

We conclude that $q(\beta \alpha)=(b a) p=f p$, and hence for any $R$-linear map $g: G \longrightarrow H$ with $q g=f p$ we have $[g]=[\beta \alpha] \in \mathfrak{F P}-\operatorname{Hom}_{R}(G, H)$ (cf. (ii) above). This finishes the proof, since we obviously have $[\beta \alpha]=[0] \in \mathfrak{F P}-\operatorname{Hom}_{R}(G, H)$.

Let $\mathfrak{F P}-\mathrm{GP}(R)$ and $\mathfrak{F P}-\mathrm{FGP}(R)$ be the full subcategories of $\mathfrak{F P}-R$-Mod, whose objects are the Gorenstein projective modules and the modules of finite Gorenstein projective dimension respectively; then, $\mathfrak{F P}-\mathrm{GP}(R)$ is a full subcategory of $\mathfrak{F P - F G P}(R)$. We note that if $G, A$ are two modules with $G$ Gorenstein projective and $A$ of finite projective dimension, then the group $\operatorname{Ext}_{R}^{1}(G, \Omega A)$ is trivial, in view of the finiteness of the projective dimension of $\Omega A$; cf. Corollary 1.5(i). It follows that any $R$-linear map $f: G \longrightarrow A$ factors as the composition of $R$-linear maps $G \longrightarrow P \stackrel{p}{\longrightarrow} A$, where $P$ is a projective module and $p$ is surjective 1 Therefore, we conclude that an $R$-linear map between Gorenstein projective modules factors through a module of finite projective dimension if and only if it factors through a projective module. Hence, the category $\mathfrak{F P}$-GP $(R)$ defined above is precisely the stable category of Gorenstein projective modules.

We observe that Lemmas 1.4(i) and 2.1 imply that there is a well-defined additive functor

$$
\mu: \mathfrak{F P}-\mathrm{FGP}(R) \longrightarrow \mathfrak{F P}-\mathrm{GP}(R),
$$

which maps a module $M$ of finite Gorenstein projective dimension to the Gorenstein projective module $G$ that appears in the short exact sequence of Proposition 1.2(ii) and the class $[f] \in \mathfrak{F P}-\operatorname{Hom}_{R}(M, N)$ of an $R$-linear map $f: M \longrightarrow N$ to the class $[g] \in \mathfrak{F P}^{-H_{o m}}(G, H)$ of any $R$-linear map $g: G \longrightarrow H$ that satisfies the condition described in Lemma 2.1(i). We can now state the main result of this section.

Theorem 2.2. The additive functor $\mu: \mathfrak{F P}-\mathrm{FGP}(R) \longrightarrow \mathfrak{F P}-\mathrm{GP}(R)$ defined above is right adjoint to the inclusion functor $\mathfrak{F P}-\mathrm{GP}(R) \hookrightarrow \mathfrak{F P}-\mathrm{FGP}(R)$.

Proof. We fix a Gorenstein projective module $G$ and let $N$ be a module of finite Gorenstein projective dimension. We also consider a short exact sequence of $R$ modules,

$$
0 \longrightarrow L \stackrel{\jmath}{\longrightarrow} H \stackrel{q}{\longrightarrow} N \longrightarrow 0
$$

\footnotetext{
${ }^{1}$ Dually, we may express $G$ as $\Omega G^{\prime}$, for some Gorenstein projective module $G^{\prime}$, and use the vanishing of the abelian group $\operatorname{Ext}_{R}^{1}\left(G^{\prime}, A\right)$ in order to factor the $R$-linear map $f: G \longrightarrow A$ as the composition of $R$-linear maps $G \stackrel{\iota}{\longrightarrow} P^{\prime} \longrightarrow A$, where $P^{\prime}$ is a projective module and $\iota$ is injective.
} 
where $\operatorname{pd}_{R} L<\infty$ and $H$ is Gorenstein projective (cf. Proposition 1.2(ii)). We note that the additive map

$$
[q]_{*}: \mathfrak{F P}-\operatorname{Hom}_{R}(G, H) \longrightarrow \mathfrak{F P}-\operatorname{Hom}_{R}(G, N)
$$

is natural in both $G$ (this is straightforward) and $N$ (this follows from Lemma 2.1). We shall establish the adjunction in the statement of the theorem by proving that the additive map (3) above is bijective. Indeed, since the module $G$ is Gorenstein

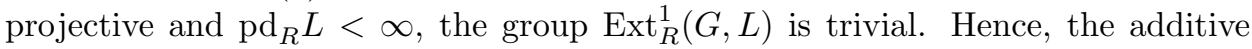
map

$$
q_{*}: \operatorname{Hom}_{R}(G, H) \longrightarrow \operatorname{Hom}_{R}(G, N)
$$

is surjective, whence the surjectivity of (3). As far as the injectivity of (3) is concerned, assume that $g: G \longrightarrow H$ is an $R$-linear map, such that $[q g]=[q][g]=$ $[q]_{*}[g]=[0] \in \mathfrak{F P}-\operatorname{Hom}_{R}(G, N)$. Then, we may consider the commutative diagram

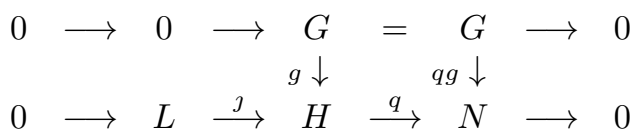

and invoke Lemma 2.1(iii) in order to conclude that $[g]=[0] \in \mathfrak{F P}-\operatorname{Hom}_{R}(G, H)$.

We shall now prove that triviality of the counit of the adjunction described in Theorem 2.2 characterizes the modules of finite projective dimension among the modules of finite Gorenstein projective dimension.

Corollary 2.3. The following conditions are equivalent for a module $N$ of finite Gorenstein projective dimension:

(i) $p d_{R} N<\infty$,

(ii) $\mathfrak{F P}-H_{o m}(G, N)=0$ for all Gorenstein projective modules $G$ and

(iii) there exists a short exact sequence of $R$-modules $0 \longrightarrow L \longrightarrow H \stackrel{q}{\longrightarrow}$ $N \longrightarrow 0$, where $p d_{R} L<\infty$ and $H$ is Gorenstein projective, such that $[q]=[0] \in$ $\mathfrak{F P}-\mathrm{Hom}_{R}(H, N)$.

Proof. The implications (i) $\rightarrow$ (ii) and (ii) $\rightarrow$ (iii) are obvious, and hence it only remains to show that (iii) $\rightarrow$ (i). To that end, we note that the adjunction isomorphism (3) established in the proof of Theorem 2.2 implies, in particular, that the additive map

$$
[q]_{*}: \mathfrak{F P}-\operatorname{Hom}_{R}(H, H) \longrightarrow \mathfrak{F P}-\operatorname{Hom}_{R}(H, N)
$$

is bijective. Since $[q]_{*}\left[1_{H}\right]=[q]\left[1_{H}\right]=\left[q 1_{H}\right]=[q]=[0] \in \mathfrak{F P}-\operatorname{Hom}_{R}(H, N)$, we conclude that $\left[1_{H}\right]=[0] \in \mathfrak{F P}-\operatorname{Hom}_{R}(H, H)$; hence, the identity map of $H$ factors through a module $A$ of finite projective dimension. It follows that $H$ is a direct summand of $A$, and hence $H$ has finite projective dimension as well. Being a Gorenstein projective module of finite projective dimension, the module $H$ is necessarily projective (cf. [18, Proposition 2.27]). It follows readily that $\operatorname{pd}_{R} N \leq$ $\operatorname{pd}_{R} L+1<\infty$, as needed.

\section{Stabilizing With Respect to Gorenstein projective modules}

Let $M, N$ be two modules. Then, the set consisting of those $R$-linear maps $f: M \longrightarrow N$ that factor through a Gorenstein projective module is a subgroup of the abelian group $\operatorname{Hom}_{R}(M, N)$. (This follows since the direct sum of two Gorenstein projective modules is Gorenstein projective as well.) We shall denote by 
$\mathfrak{G P}-\operatorname{Hom}_{R}(M, N)$ the corresponding quotient group and let $[f]=[f]_{\mathfrak{G P}}$ be the class of any $R$-linear map $f \in \operatorname{Hom}_{R}(M, N)$ therein. We note that for any three modules $M, N$ and $L$ the composition of $R$-linear maps induces a well-defined biadditive map

$$
\mathfrak{G P}-\operatorname{Hom}_{R}(N, L) \times \mathfrak{G P}-\operatorname{Hom}_{R}(M, N) \longrightarrow \mathfrak{G P}-\operatorname{Hom}_{R}(M, L) .
$$

We shall denote by $\mathfrak{G P}$ - $R$-Mod the category whose objects are all modules and whose morphism sets are given by the abelian groups $\mathfrak{G P}-\operatorname{Hom}_{R}(M, N)$ (with the composition of morphisms induced by the composition of $R$-linear maps). Then, Lemma 1.4 implies that the modules $K$ and $A$, which are defined for any module $M$ of finite Gorenstein projective dimension in Proposition 1.2(ii) and (iii), are uniquely determined up to isomorphism as objects of the category $\mathfrak{G P}$ - $R$-Mod.

Lemma 3.1. Let $f: M \longrightarrow N$ be an $R$-linear map, where $M, N$ are two modules of finite Gorenstein projective dimension. We also consider two exact sequences of $R$-modules,

$$
0 \longrightarrow M \stackrel{\iota}{\longrightarrow} A \stackrel{p}{\longrightarrow} G^{\prime} \longrightarrow 0 \text { and } 0 \longrightarrow N \stackrel{\jmath}{\longrightarrow} B \stackrel{q}{\longrightarrow} H^{\prime} \longrightarrow 0,
$$

where $A, B$ have finite projective dimension and $G^{\prime}, H^{\prime}$ are Gorenstein projective. Then:

(i) There exists an R-linear map $g: A \longrightarrow B$, such that $g \iota=\jmath f$.

(ii) If $g, g^{\prime}: A \longrightarrow B$ are two $R$-linear maps with $g \iota=\jmath f$ and $g^{\prime} \iota=\jmath f$, then $[g]=\left[g^{\prime}\right] \in \mathfrak{G P}-H_{0} m_{R}(A, B)$.

(iii) If $[f]=[0] \in \mathfrak{G P}-H_{R}(M, N)$ and $g: A \longrightarrow B$ is an $R$-linear map such that $g \iota=\jmath f$, then $[g]=[0] \in \mathfrak{G P}-H_{0} m_{R}(A, B)$.

Proof. (i) Since $G^{\prime}$ is Gorenstein projective and $\operatorname{pd}_{R} B<\infty$, the group $\operatorname{Ext}_{R}^{1}\left(G^{\prime}, B\right)$ is trivial, and hence the additive map $\iota^{*}: \operatorname{Hom}_{R}(A, B) \longrightarrow \operatorname{Hom}_{R}(M, B)$ is surjective. Therefore, there exists an $R$-linear map $g: A \longrightarrow B$ such that $\jmath f=\iota^{*}(g)$, as needed.

(ii) Let $g, g^{\prime}: A \longrightarrow B$ be two $R$-linear maps with $g \iota=\jmath f$ and $g^{\prime} \iota=\jmath f$ :

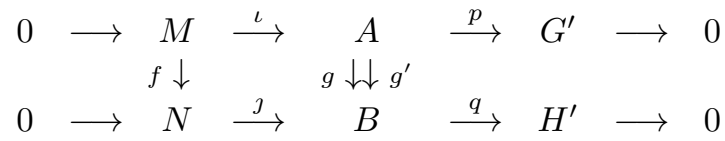

Then, $\left(g^{\prime}-g\right) \iota=g^{\prime} \iota-g \iota=\jmath f-\jmath f=0$, and hence there exists an $R$-linear map $h: G^{\prime} \longrightarrow B$, such that $g^{\prime}-g=h p$. Since $G^{\prime}$ is Gorenstein projective, we conclude that $[g]=\left[g^{\prime}\right] \in \mathfrak{G P}-\operatorname{Hom}_{R}(A, B)$.

(iii) Assume that $f$ factors as the composition of two $R$-linear maps $M \stackrel{a}{\longrightarrow} \Gamma \stackrel{b}{\longrightarrow}$ $N$, where the module $\Gamma$ is Gorenstein projective. Let

$$
0 \longrightarrow \Gamma \stackrel{k}{\longrightarrow} P \longrightarrow \Gamma^{\prime} \longrightarrow 0
$$

be a short exact sequence of $R$-modules with $P$ projective and $\Gamma^{\prime}$ Gorenstein projective. We may construct such an exact sequence by considering a totally acyclic complex of projective modules with $\Gamma$ as a syzygy (cf. Lemma 1.1). Then, invoking 
(i) above, we may find $R$-linear maps $\alpha: A \longrightarrow P$ and $\beta: P \longrightarrow B$, such that $\alpha \iota=k a$ and $\beta k=\jmath b$ :

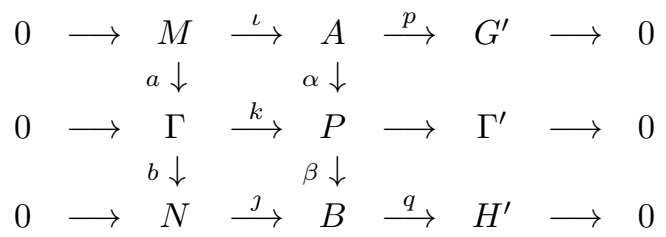

We conclude that $(\beta \alpha) \iota=\jmath(b a)=\jmath f$, and hence for any $R$-linear map $g: A \longrightarrow B$ with $g \iota=\jmath f$ we have $[g]=[\beta \alpha] \in \mathfrak{G P}-\operatorname{Hom}_{R}(A, B)$ (cf. (ii) above). This finishes the proof, since we obviously have $[\beta \alpha]=[0] \in \mathfrak{G P}-\operatorname{Hom}_{R}(A, B)$.

Let $\mathfrak{G P}-\mathrm{FP}(R)$ and $\mathfrak{G P}-\mathrm{FGP}(R)$ be the full subcategories of $\mathfrak{G P}-R$-Mod whose objects are the modules of finite projective and finite Gorenstein projective dimension respectively; then, $\mathfrak{G P}-\mathrm{FP}(R)$ is a full subcategory of $\mathfrak{G P}-\mathrm{FGP}(R)$. As we have noted in the discussion following Lemma 2.1, any $R$-linear map $f: G \longrightarrow A$, where $G$ is Gorenstein projective and $A$ has finite projective dimension, factors through a projective module. It follows that an $R$-linear map between modules of finite projective dimension factors through a Gorenstein projective module if and only if it factors through a projective module. Hence, the category $\mathfrak{G P}-\mathrm{FP}(R)$ defined above is precisely the stable category of modules of finite projective dimension.

We observe that Lemmas 1.4(ii) and 3.1 imply that there is a well-defined additive functor

$$
\nu: \mathfrak{G P}-\mathrm{FGP}(R) \longrightarrow \mathfrak{G P}-\mathrm{FP}(R)
$$

which maps a module $M$ of finite Gorenstein projective dimension to the module $A$ of finite projective dimension that appears in the short exact sequence of Proposition 1.2(iii) and the class $[f] \in \mathfrak{G P}-\operatorname{Hom}_{R}(M, N)$ of an $R$-linear map $f: M \longrightarrow N$ to the class $[g] \in \mathfrak{G P}-\operatorname{Hom}_{R}(A, B)$ of any $R$-linear map $g: A \longrightarrow B$ that satisfies the condition described in Lemma 3.1(i). We can now state the main result of this section.

Theorem 3.2. The additive functor $\nu: \mathfrak{G P}-\mathrm{FGP}(R) \longrightarrow \mathfrak{G P}-\mathrm{FP}(R)$ defined above is left adjoint to the inclusion functor $\mathfrak{G P}-\mathrm{FP}(R) \hookrightarrow \mathfrak{G P}-\mathrm{FGP}(R)$.

Proof. We fix a module $B$ of finite projective dimension and let $M$ be a module of finite Gorenstein projective dimension. We also consider a short exact sequence of $R$-modules

$$
0 \longrightarrow M \stackrel{\iota}{\longrightarrow} A \stackrel{p}{\longrightarrow} G^{\prime} \longrightarrow 0,
$$

where $\operatorname{pd}_{R} A<\infty$ and $G^{\prime}$ is Gorenstein projective (cf. Proposition 1.2(iii)). We note that the additive map

$$
[\iota]^{*}: \mathfrak{G P}-\operatorname{Hom}_{R}(A, B) \longrightarrow \mathfrak{G P}-\operatorname{Hom}_{R}(M, B)
$$

is natural in both $B$ (this is straightforward) and $M$ (this follows from Lemma 3.1). We shall establish the adjunction in the statement of the theorem by proving that 
the additive map (4) above is bijective. Indeed, since the module $G^{\prime}$ is Gorenstein projective and $\operatorname{pd}_{R} B<\infty$, the group $\operatorname{Ext}_{R}^{1}\left(G^{\prime}, B\right)$ is trivial. Hence, the additive map

$$
\iota^{*}: \operatorname{Hom}_{R}(A, B) \longrightarrow \operatorname{Hom}_{R}(M, B)
$$

is surjective, whence the surjectivity of (4). As far as the injectivity of (4) is concerned, assume that $g: A \longrightarrow B$ is an $R$-linear map, such that $[g \iota]=[g][\iota]=$ $[\iota]^{*}[g]=[0] \in \mathfrak{G P}-\operatorname{Hom}_{R}(M, B)$. Then, we may consider the commutative diagram

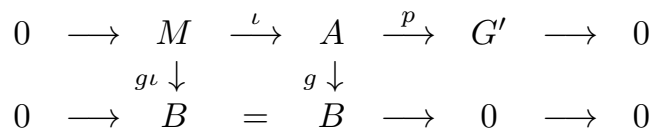

and invoke Lemma 3.1(iii), in order to conclude that $[g]=[0] \in \mathfrak{G P}-\operatorname{Hom}_{R}(A, B)$.

We shall now prove that the triviality of the unit of the adjunction described in Theorem 3.2 characterizes the Gorenstein projective modules among the modules of finite Gorenstein projective dimension.

Corollary 3.3. The following conditions are equivalent for a module $M$ of finite Gorenstein projective dimension:

(i) $M$ is Gorenstein projective,

(ii) $\mathfrak{G P}-H_{0} m_{R}(M, B)=0$ for all modules $B$ with $p d_{R} B<\infty$ and

(iii) there exists a short exact sequence of $R$-modules $0 \longrightarrow M \stackrel{\iota}{\longrightarrow} A \longrightarrow$ $G^{\prime} \longrightarrow 0$, where $p d_{R} A<\infty$ and $G^{\prime}$ is Gorenstein projective, such that $[\iota]=[0] \in$ $\mathfrak{G P}-\mathrm{Hom}_{R}(M, A)$.

Proof. The implications (i) $\rightarrow$ (ii) and (ii) $\rightarrow$ (iii) are obvious, and hence it only remains to show that (iii) $\rightarrow$ (i). To that end, we note that the adjunction isomorphism (4) established in the proof of Theorem 3.2 implies, in particular, that the additive map

$$
[\iota]^{*}: \mathfrak{G P}-\operatorname{Hom}_{R}(A, A) \longrightarrow \mathfrak{G P}-\operatorname{Hom}_{R}(M, A)
$$

is bijective. Since $[\iota]^{*}\left[1_{A}\right]=\left[1_{A}\right][\iota]=\left[1_{A} \iota\right]=[\iota]=[0] \in \mathfrak{G P}-\operatorname{Hom}_{R}(M, A)$, we conclude that $\left[1_{A}\right]=[0] \in \mathfrak{G P}-\operatorname{Hom}_{R}(A, A)$; hence, the identity map of $A$ factors through a Gorenstein projective module $G$. It follows that $A$ is a direct summand of $G$ and hence $A$ is Gorenstein projective (cf. [18, Theorem 2.5]). Being a Gorenstein projective module of finite projective dimension, the module $A$ is necessarily projective (cf. [18, Proposition 2.27]). Then, Lemma 1.1 implies that $M$ is Gorenstein projective, as needed.

\section{Finiteness of Gorenstein projective Dimension AND COMPLETE COHOMOLOGY}

Farrell has generalized in [15] the classical Tate cohomology theory of finite groups (cf. [9, Chapter XII] and [8, Chapter VI]) to the class of groups with finite virtual cohomological dimension. More generally, the existence of terminal com- 
pletions of the ordinary Ext functors has been studied by Gedrich and Gruenberg in [16. Mislin developed in 21] a generalization of the Tate-Farrell cohomology that may be defined over any ring $R$. Using an approach that involves satellites, he defined for any module $M$ complete cohomology functors $\widehat{\operatorname{Ext}}_{R}^{*}\left(M,_{-}\right)$and a natural transformation $\operatorname{Ext}_{R}^{*}\left(M,_{-}\right) \longrightarrow \widehat{\operatorname{Ext}}_{R}^{*}\left(M,_{-}\right)$as the projective completion of the ordinary Ext functors $\operatorname{Ext}_{R}^{*}\left(M,_{-}\right)$. Mislin's approach was heavily influenced by Gedrich and Gruenberg's theory of terminal completions. As an immediate consequence of the definition, we note that the complete cohomology groups $\widehat{\operatorname{Ext}}_{R}^{*}(M, N)$ vanish if $\operatorname{pd}_{R} N<\infty$. Equivalent definitions of the complete cohomology functors have been independently formulated by Vogel in [17] (using a hypercohomology approach) and by Benson and Carlson in [7] (using projective resolutions). Using the approach by Benson and Carlson, it follows that the elements in the kernel of the canonical map $\operatorname{Hom}_{R}(M, N) \longrightarrow \widehat{\operatorname{Ext}}_{R}^{0}(M, N)$ are those $R$-linear maps $f: M \longrightarrow N$ which are such that the $R$-linear map $\Omega^{n} f: \Omega^{n} M \longrightarrow \Omega^{n} N$ induced by $f$ between the $n$-th syzygy modules of $M$ and $N$ factors through a projective module for $n \gg 0$. For a thorough discussion of complete cohomology, the reader may also consult [5].

Assume that $M$ is a module of finite Gorenstein projective dimension. Then, $M$ admits a strong complete projective resolution, i.e. there exists a totally acyclic complex of projective modules $P_{*}$, which coincides with an ordinary projective resolution $Q_{*}$ of $M$ in sufficiently large degrees. If $\tau_{*}: P_{*} \longrightarrow Q_{*}$ is a chain map which is the identity in sufficiently large degrees, then the complete cohomology functors $\widehat{\operatorname{Ext}}_{R}^{*}\left(M,{ }_{-}\right)$may be computed as the cohomology groups of the complex $\operatorname{Hom}_{R}\left(P_{*},-\right)$, whereas the canonical map $\operatorname{Ext}_{R}^{*}\left(M,_{-}\right) \longrightarrow \widehat{\operatorname{Ext}}_{R}^{*}\left(M,_{-}\right)$is that induced by the chain map $\tau_{*}$. This is an immediate consequence of Mislin's satellite approach (cf. [21, Lemma 2.4]). In particular, since the strong complete projective resolution of $M$ may be chosen to coincide with an ordinary projective resolution of $M$ in degrees $\geq \operatorname{Gpd}_{R} M$, it follows that the canonical map $\operatorname{Ext}_{R}^{n}(M, N) \longrightarrow$ $\widehat{\operatorname{Ext}}_{R}^{n}(M, N)$ is bijective if $n>\operatorname{Gpd}_{R} M$ and surjective if $n=\operatorname{Gpd}_{R} M$.

Remark 4.1. Let $M$ be a module of finite injective dimension. Then, as shown by Holm in [19, Theorem 2.2], we have an equality $\operatorname{Gpd}_{R} M=\operatorname{pd}_{R} M$. The key point in his proof consists in showing that the finiteness of $\operatorname{Gpd}_{R} M$ implies that $\operatorname{pd}_{R} M$ is finite as well. An alternative proof of the latter implication may be obtained by using complete cohomology as follows: In view of the finiteness of $\operatorname{Gpd}_{R} M$, the module $M$ admits a strong complete projective resolution $P_{*}$ and the complete cohomology functors $\widehat{\operatorname{Ext}}_{R}^{*}\left(M,{ }_{-}\right)$may be computed as the cohomology groups of the complex $\operatorname{Hom}_{R}\left(P_{*},{ }_{-}\right)$. Since $P_{*}$ is an acyclic complex of projective modules, the complex of abelian groups $\operatorname{Hom}_{R}\left(P_{*}, L\right)$ is acyclic for any module $L$ of finite injective dimension. In particular, the complex $\operatorname{Hom}_{R}\left(P_{*}, M\right)$ is acyclic and hence $\widehat{\operatorname{Ext}}_{R}^{*}(M, M)=0$. As shown by Kropholler in [20, $\left.\S 4.2\right]$, one may use the BensonCarlson approach to complete cohomology in order to show that the vanishing of the group $\widehat{\operatorname{Ext}}_{R}^{0}(M, M)$ implies that the projective dimension of $M$ is finite, as needed.

The next result shows that the conditions on the finiteness of the projective dimension of the modules $K$ and $A$ that appear in the short exact sequences in 
Proposition 1.2(ii) and (iii) may be relaxed to the assertion that certain elements of complete cohomology groups vanish.

Theorem 4.2. The following conditions are equivalent for a module $M$ :

(i) $\operatorname{Gpd}_{R} M<\infty$,

(ii) there exists a short exact sequence of $R$-modules $0 \longrightarrow K \stackrel{\iota}{\longrightarrow} G \stackrel{p}{\longrightarrow} M \longrightarrow$ 0 , where $G$ is Gorenstein projective, such that the image of the classifying element $\xi \in \operatorname{Ext}_{R}^{1}(M, K)$ vanishes in the group $\widehat{\operatorname{Ext}}_{R}^{1}(M, K)$, and

(iii) there exists a short exact sequence of $R$-modules $0 \longrightarrow M \stackrel{3}{\longrightarrow} A \stackrel{q}{\longrightarrow} G^{\prime} \longrightarrow$ 0 , where $G^{\prime}$ is Gorenstein projective, such that the image of $\mathrm{\jmath} \in \operatorname{Hom}_{R}(M, A)$ vanishes in the group $\widehat{\operatorname{Ext}}_{R}^{0}(M, A)$.

Proof. (i) $\rightarrow$ (ii): Our hypothesis implies the existence of a short exact sequence of $R$-modules,

$$
0 \longrightarrow K \stackrel{\iota}{\longrightarrow} G \stackrel{p}{\longrightarrow} M \longrightarrow 0,
$$

where $G$ is Gorenstein projective and $\operatorname{pd}_{R} K<\infty$ (cf. Proposition 1.2(ii)). Then, $\widehat{\operatorname{Ext}}_{R}^{1}(M, K)$ is the trivial group, and hence the image of the classifying element $\xi$ vanishes therein.

(ii) $\rightarrow$ (i): We consider the commutative diagram with exact rows:

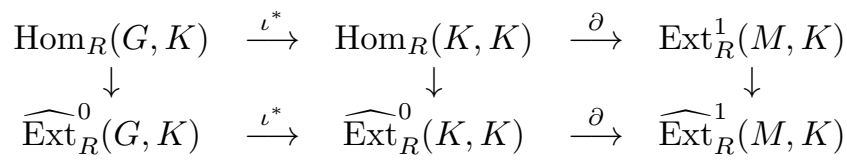

Since the module $G$ is Gorenstein projective, the additive map $\operatorname{Hom}_{R}(G, K) \longrightarrow$ $\widehat{\operatorname{Ext}}_{R}^{0}(G, K)$ is surjective. Then, our assumption about the vanishing of the image $[\xi]$ of $\xi=\partial\left(1_{K}\right)$ in the group $\widehat{\operatorname{Ext}}_{R}^{1}(M, K)$ is easily seen to imply the existence of an $R$-linear map $f \in \operatorname{Hom}_{R}(G, K)$, such that $[f \iota]=\left[1_{K}\right] \in \widehat{\operatorname{Ext}}_{R}^{0}(K, K)$. Therefore, if we denote by $g$ the endomorphism $1_{K}-f \iota$ of $K$, then the induced endomorphism $\Omega^{n} g$ of $\Omega^{n} K$ factors through a projective module for $n \gg 0$. On the other hand, it is clear that the endomorphism $\Omega^{n}(f \iota)=\left(\Omega^{n} f\right)\left(\Omega^{n} \iota\right)$ of $\Omega^{n} K$ factors through $\Omega^{n} G$ for all $n$. Since $G$ is Gorenstein projective, this is also the case for all of the $\Omega^{n} G^{\prime}$ s (cf. Lemma 1.1). With any projective module being Gorenstein projective, it follows that $\Omega^{n} 1_{K}=\Omega^{n}(g+f \iota)=\Omega^{n} g+\Omega^{n}(f \iota)$ factors through a Gorenstein projective module for $n \gg 0$. Since $\Omega^{n} 1_{K}$ is the identity map of $\Omega^{n} K$, we conclude that $\Omega^{n} K$ is a direct summand of a Gorenstein projective module and hence is itself Gorenstein projective for $n \gg 0$ (cf. [18, Theorem 2.5]). It follows that $\operatorname{Gpd}_{R} K<\infty$, and the short exact sequence

$$
0 \longrightarrow K \stackrel{\iota}{\longrightarrow} G \stackrel{p}{\longrightarrow} M \longrightarrow 0
$$

then implies that $\operatorname{Gpd}_{R} M \leq \operatorname{Gpd}_{R} K+1<\infty$.

(i) $\rightarrow$ (iii): Our hypothesis implies the existence of a short exact sequence of $R$ modules,

$$
0 \longrightarrow M \stackrel{3}{\longrightarrow} A \stackrel{q}{\longrightarrow} G^{\prime} \longrightarrow 0,
$$

where $G^{\prime}$ is Gorenstein projective and $\operatorname{pd}_{R} A<\infty$ (cf. Proposition 1.2(iii)). Then, $\widehat{\operatorname{Ext}}_{R}^{0}(M, A)$ is the trivial group, and hence the image of $\jmath$ vanishes therein. 
(iii) $\rightarrow$ (i): We consider the commutative diagram with exact rows:

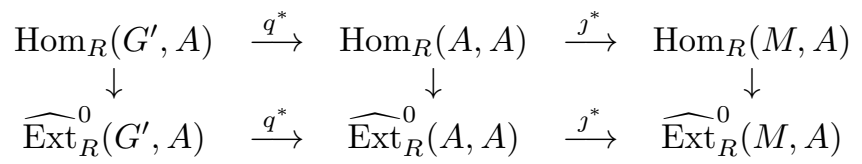

Since the module $G^{\prime}$ is Gorenstein projective, the additive map $\operatorname{Hom}_{R}\left(G^{\prime}, A\right) \longrightarrow$ $\widehat{\operatorname{Ext}}_{R}^{0}\left(G^{\prime}, A\right)$ is surjective. Then, our assumption about the vanishing of the image [j] of $\jmath=\jmath^{*}\left(1_{A}\right)$ in the group $\widehat{\operatorname{Ext}}_{R}(M, A)$ is easily seen to imply the existence of an $R$-linear map $f \in \operatorname{Hom}_{R}\left(G^{\prime}, A\right)$, such that $[f q]=\left[1_{A}\right] \in \widehat{\operatorname{Ext}}_{R}^{0}(A, A)$. Therefore, if we denote by $g$ the endomorphism $1_{A}-f q$ of $A$, then the induced endomorphism $\Omega^{n} g$ of $\Omega^{n} A$ factors through a projective module for $n \gg 0$. On the other hand, it is clear that the endomorphism $\Omega^{n}(f q)=\left(\Omega^{n} f\right)\left(\Omega^{n} q\right)$ of $\Omega^{n} A$ factors through $\Omega^{n} G^{\prime}$ for all $n$. Since $G^{\prime}$ is Gorenstein projective, this is also the case for all of the $\Omega^{n} G^{\prime}$ s (cf. Lemma 1.1). With any projective module being Gorenstein projective, it follows that $\Omega^{n} 1_{A}=\Omega^{n}(g+f q)=\Omega^{n} g+\Omega^{n}(f q)$ factors through a Gorenstein projective module for $n \gg 0$. Since $\Omega^{n} 1_{A}$ is the identity map of $\Omega^{n} A$, we conclude that $\Omega^{n} A$ is a direct summand of a Gorenstein projective module and hence is itself Gorenstein projective for $n \gg 0$ (cf. [18, Theorem 2.5]). It follows that $\operatorname{Gpd}_{R} A<\infty$, and the short exact sequence

$$
0 \longrightarrow M \stackrel{\jmath}{\longrightarrow} A \stackrel{q}{\longrightarrow} G^{\prime} \longrightarrow 0
$$

then implies that $\operatorname{Gpd}_{R} M<\infty$ (cf. [18, Theorem 2.24]).

Remark 4.3. A short exact sequence of $R$-modules $0 \longrightarrow K \longrightarrow G \longrightarrow M \longrightarrow$ 0 induces an $R$-linear map $f: \Omega M \longrightarrow K$ whose image under the connecting homomorphism

$$
\partial: \operatorname{Hom}_{R}(\Omega M, K) \longrightarrow \operatorname{Ext}_{R}^{1}(M, K)
$$

is the classifying element $\xi \in \operatorname{Ext}_{R}^{1}(M, K)$. The $R$-linear map $f$ is unique up to linear maps that factor through projective modules, and hence its image $[f]$ in the complete cohomology group $\widehat{\operatorname{Ext}}_{R}^{0}(\Omega M, K)$ depends only upon the given short exact sequence. Moreover, the image of $[f]$ under the connecting homomorphism

$$
\partial: \widehat{\operatorname{Ext}}_{R}^{0}(\Omega M, K) \longrightarrow \widehat{\operatorname{Ext}}_{R}^{1}(M, K)
$$

is the image $[\xi]$ of the classifying element $\xi$ in the group $\widehat{\operatorname{Ext}}_{R}^{1}(M, K)$; this follows from the commutativity of the diagram

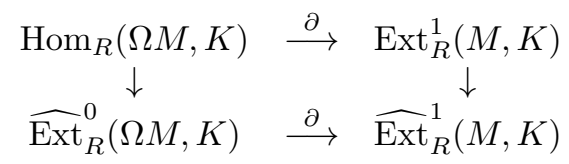

The connecting homomorphism (5) is evidently bijective; this follows since the complete cohomology groups are trivial in the case where the first argument is a projective module. Hence, the assumption about the vanishing of the element 
$[\xi] \in \widehat{\operatorname{Ext}}_{R}^{1}(M, K)$, which appears in Theorem 4.2(ii), is equivalent to the vanishing of the element $[f] \in \widehat{\operatorname{Ext}}_{R}^{0}(\Omega M, K)$.

\section{Modules of Finite Gorenstein injective Dimension}

The class of Gorenstein injective modules was defined by Enochs and Jenda in [14] by using an approach dual to that defining Gorenstein projective modules. Modules of finite Gorenstein injective dimension are then defined using resolutions by Gorenstein injective modules. The arguments that were employed in order to establish the properties of modules of finite Gorenstein projective dimension in the previous sections can be easily dualized and lead to analogous results concerning modules of finite Gorenstein injective dimension. In fact, as shown in 18, Theorem 2.15] and [11, Lemma 2.18], the following conditions are equivalent for a module $M$ and a non-negative integer $n$ :

(i) $\operatorname{Gid}_{R} M \leq n$,

(ii) there exists a short exact sequence of $R$-modules $0 \longrightarrow M \longrightarrow Q \longrightarrow L \longrightarrow$ 0 , where $Q$ is Gorenstein injective and $\operatorname{id}_{R} L \leq n-1$ (if $n=0$, this is understood to mean $L=0$ ), and

(iii) there exists a short exact sequence of $R$-modules $0 \longrightarrow Q^{\prime} \longrightarrow B \longrightarrow M \longrightarrow$ 0 , where $Q^{\prime}$ is Gorenstein injective and $\operatorname{id}_{R} B \leq n$.

As with Gorenstein projective dimension, there is a weak form of uniqueness (à la Schanuel) for the short exact sequences in (ii) and (iii) above. This uniqueness may be used in order to turn the assignments $M \mapsto Q$ and $M \mapsto B$ into functors between subcategories of appropriate stabilizations of the category of $R$-modules. These functors are adjoints of suitable inclusion functors, and hence the exact sequences above may be considered as approximations of $M$, in the sense of Auslander and Buchweitz. We leave it to the reader to complete the details by providing the dual arguments to those used in sections 1,2 and 3 above.

In analogy with Theorem 4.2, we may characterize the finiteness of the Gorenstein injective dimension of $M$ by relaxing the hypotheses on the finiteness of the injective dimension of the modules $L$ and $B$ which appear in the exact sequences of (ii) and (iii) above, in a way that involves Nucinkis' injective complete cohomology Ext (cf. [22]). This cohomology theory may be defined by the duals of the three approaches that define the (projective) complete cohomology $\widehat{\text { Ext. }}$. The complete cohomology groups $\widetilde{\operatorname{Ext}}_{R}^{*}(M, N)$ vanish if $M$ is a module of finite injective dimension. It is interesting to note that the complete cohomology functors $\widehat{\text { Ext }}$ and $\widetilde{\mathrm{Ext}}$ are naturally isomorphic if and only if the ring $R$ has finite left Gorenstein global dimension (cf. [22, Theorem 5.2]). The proof of the following result proceeds along the same lines as that of Theorem 4.2 by using the dual arguments; the details are left to the reader.

Theorem 5.1. The following conditions are equivalent for a module $M$ :

(i) $\operatorname{Gid}_{R} M<\infty$,

(ii) there exists a short exact sequence of $R$-modules $0 \longrightarrow M \longrightarrow Q \longrightarrow L \longrightarrow 0$, where $Q$ is Gorenstein injective, such that the image of the classifying element $\xi \in \operatorname{Ext}_{R}^{1}(L, M)$ vanishes in the group $\widetilde{\operatorname{Ext}_{R}^{1}}(L, M)$, and

(iii) there exists a short exact sequence of $R$-modules $0 \longrightarrow Q^{\prime} \longrightarrow B \stackrel{q}{\longrightarrow}$ $M \longrightarrow 0$, where $Q^{\prime}$ is Gorenstein injective, such that the image of $q \in \operatorname{Hom}_{R}(B, M)$ vanishes in the group $\widetilde{\operatorname{Ext}_{R}}(B, M)$. 


\section{The SPECial Case of Group Rings}

In this final section, we consider a commutative $\operatorname{ring} k$, a group $G$ and let $R=k G$ be the associated group algebra. Then, using the diagonal action of the group $G$, the tensor product $2 M \otimes N$ of two $k G$-modules is also a $k G$-module. Moreover, if $M$ is a projective $k G$-module and $N$ is a $k G$-module which is $k$-projective, then the $k G$-module $M \otimes N$ is projective as well (cf. [8, Corollary 5.7]). Therefore, if $P_{*} \longrightarrow M \longrightarrow 0$ is a projective resolution of a $k G$-module $M$ and $N$ is a $k$-projective $k G$-module, then $P_{*} \otimes N \longrightarrow M \otimes N \longrightarrow 0$ is a projective resolution of the $k G$ module $M \otimes N$. It follows that we then have an equality $\Omega^{n}(M \otimes N)=\Omega^{n} M \otimes N$ for all $n \geq 0$, whereas $\operatorname{pd}_{k G}(M \otimes N) \leq \operatorname{pd}_{k G} M$.

Lemma 6.1. Let $k$ be a commutative ring and consider a direct system of $k$-modules and linear maps,

$$
P_{0} \stackrel{f_{0}}{\longrightarrow} P_{1} \stackrel{f_{1}}{\longrightarrow} \cdots \stackrel{f_{n-1}}{\longrightarrow} P_{n} \stackrel{f_{n}}{\longrightarrow} \cdots .
$$

We assume that $f_{n}$ is a split monomorphism for all $n \geq 0$ and define the $k$-module $P$ to be the direct limit of the system. Then:

(i) the canonical map $P_{0} \longrightarrow P$ is a split monomorphism and

(ii) if $P_{n}$ is projective for all $n$, then $P$ is projective as well.

Proof. In view of our hypothesis about $f_{n}$, we can assume that there exists a $k$ module $Q_{n+1}$, such that $P_{n+1}=P_{n} \oplus Q_{n+1}$ and the structural map $f_{n}: P_{n} \longrightarrow$ $P_{n+1}$ is the map $x \mapsto(x, 0), x \in P_{n}$, for all $n \geq 0$. Then, we have $P_{n}=P_{0} \oplus$ $\left(\bigoplus_{i=1}^{n} Q_{i}\right)$ for all $n \geq 0$, and hence we conclude that $P \simeq P_{0} \oplus\left(\bigoplus_{i=1}^{\infty} Q_{i}\right)$. The validity of (i) is then clear. As far as (ii) is concerned, we note that the projectivity of the $P_{n}$ 's implies that all of the $Q_{n}$ 's are projective modules; hence, $P$ is projective as well.

Proposition 6.2. Let $k$ be a commutative ring and consider a $k$-module $A$ and $a$ split monomorphism of $k$-modules $\iota: k \longrightarrow A$. We also consider the tensor powers $A^{\otimes n}, n \geq 1$, and define the direct system of $k$-modules and homomorphisms,

$$
k \longrightarrow A \longrightarrow A^{\otimes 2} \longrightarrow \cdots \longrightarrow A^{\otimes n} \longrightarrow \cdots,
$$

whose structural maps $A^{\otimes n}=k \otimes A^{\otimes n} \longrightarrow A \otimes A^{\otimes n}=A^{\otimes n+1}$ are the linear maps $\iota \otimes 1_{A}^{\otimes n}$ for all $n \geq 0$. Let $A^{\prime}$ be the direct limit of this direct system. Then:

(i) the canonical map $\iota^{\prime}: k \longrightarrow A^{\prime}$ is a split monomorphism and

(ii) if the module $A$ is projective, then $A^{\prime}$ is projective as well.

Proof. This is an immediate consequence of Lemma 6.1, since our assumption that $\iota$ is a split monomorphism implies that the structural maps $A^{\otimes n} \longrightarrow A^{\otimes n+1}$ are split monomorphisms, whereas the projectivity of $A$ implies that the tensor powers $A^{\otimes n}$ are projective modules for all $n \geq 0$.

Gedrich and Gruenberg introduced in [16] the invariants spli $k G$ and silp $k G$ as the supremum of the projective lengths of injective $k G$-modules and the supremum of the injective lengths of projective $k G$-modules respectively. The finiteness of these invariants is closely related to the existence of strong complete (projective or injective) resolutions of $k G$-modules, as shown in [16, §4]. If $k$ is a Noetherian ring of finite self-injective dimension, then we always have an equality spli $k G=\operatorname{silp} k G$

\footnotetext{
${ }^{2}$ All tensor products in this section will be understood to be over $k$.
} 
(cf. [13, Theorem 4.4]). In the proof of the next result, we shall use the following simple observation.

Observation 6.3. Let $k$ be a commutative ring of finite global dimension and consider a group $G$. Then, all Gorenstein projective $k G$-modules are $k$-projective. Indeed, let $M$ be a Gorenstein projective $k G$-module. Then, considering a totally acyclic complex of projective $k G$-modules that has $M$ as a syzygy, we conclude that for any non-negative integer $n$ there exists an exact sequence of $k G$-modules,

$$
0 \longrightarrow M \longrightarrow P_{1} \longrightarrow \cdots \longrightarrow P_{n} \longrightarrow N \longrightarrow 0,
$$

where $P_{i}$ is projective for all $i=1, \ldots, n$ (and $N$ is Gorenstein projective). Since any projective $k G$-module is a projective $k$-module, we may let $n$ be the global dimension of the ring $k$ and conclude that $M=\Omega^{n} N$ is $k$-projective, as needed.

Theorem 6.4. Let $k$ be a commutative Noetherian ring of finite global dimension. Then, the following conditions are equivalent for a group $G$ :

(i) $\operatorname{Gpd}_{k G} M<\infty$ for any $k G$-module $M$,

(ii) $\operatorname{Gcd}_{k} G<\infty$,

(iii) there exists a short exact sequence of $k G$-modules $0 \longrightarrow k \longrightarrow A \longrightarrow \bar{A} \longrightarrow$ 0 , where $\operatorname{pd}_{k G} A<\infty$ and $\bar{A}$ is Gorenstein projective,

(iv) there exists a $k$-split monomorphism of $k G$-modules $k \longrightarrow A$, where $A$ is $k$-projective and $p d_{k G} A<\infty$,

(v) there exists a $k$-split monomorphism of $k G$-modules $\iota: k \longrightarrow A$, where $A$ is $k$-projective, such that the image of $\iota \in \operatorname{Hom}_{k G}(k, A)=H^{0}(G, A)$ vanishes in the group $\widehat{\operatorname{Ext}}_{k G}^{0}(k, A)=\widehat{H}^{0}(G, A)$, and

(vi) $\operatorname{splik} G=\operatorname{silp} k G<\infty$.

Proof. The implication (i) $\rightarrow$ (ii) is obvious, since $\operatorname{Gcd}_{k} G=\operatorname{Gpd}_{k G} k$, whereas the implication (ii) $\rightarrow$ (iii) follows from Proposition 1.2.

(iii) $\rightarrow$ (iv): As we noted in Observation 6.3 above, any Gorenstein projective $k G$-module is $k$-projective. In particular, a short exact sequence of $k G$-modules as in (iii) is necessarily $k$-split and the $k G$-module $A$ therein is $k$-projective.

(iv) $\rightarrow(\mathrm{v})$ : This is clear since the assumption that the $k G$-module $A$ has finite projective dimension implies that the complete cohomology group $\widehat{\operatorname{Ext}}_{k G}^{0}(k, A)=$ $\widehat{H}^{0}(G, A)$ is trivial.

(v) $\rightarrow$ (iv): Let $P_{*} \longrightarrow k \longrightarrow 0$ be a projective resolution of the trivial $k G$-module $k$. For notational simplicity, we shall denote by $K_{i}, i \geq 0$, the corresponding syzygy modules of $k$ (i.e. we let $K_{i}=\Omega^{i} k, i \geq 0$ ). Then, $P_{*} \otimes A \longrightarrow k \otimes A \longrightarrow 0$ is a projective resolution of $k \otimes A=A$ and $K_{i} \otimes A, i \geq 0$, are the corresponding syzygy modules of $A$. A lifting of the $k G$-linear map $\iota$ is provided by the chain map $1 \otimes \iota: P_{*} \longrightarrow P_{*} \otimes A$. Using the Benson-Carlson approach to complete cohomology, we conclude that the vanishing of the image of $\iota$ under the canonical map $H^{0}(G, A) \longrightarrow \widehat{H}^{0}(G, A)$ implies the existence of a non-negative integer $s$, which is such that the map $1 \otimes \iota: K_{s} \longrightarrow K_{s} \otimes A$ factors through a projective $k G$-module $P$. We note that for any $k G$-module $B$ the map $1 \otimes \iota \otimes 1: K_{s} \otimes B \longrightarrow$ $K_{s} \otimes A \otimes B$ factors through the $k G$-module $P \otimes B$.

We now consider the direct system of $k G$-modules $\left(A^{\otimes n}\right)_{n}$, with structural maps $A^{\otimes n} \longrightarrow A^{\otimes n+1}$ given by $\iota \otimes 1_{A^{\otimes n}}$ for all $n \geq 0$, and let $A^{\prime}$ be its direct limit. Then, Proposition 6.2 implies that the $k G$-module $A^{\prime}$ is $k$-projective and the natural $k G$ linear map $\iota^{\prime}: k \longrightarrow A^{\prime}$ is a $k$-split monomorphism. In view of the very definition 
of $A^{\prime}$, it follows that there exists an isomorphism of $k G$-modules $\sigma: A \otimes A^{\prime} \longrightarrow A^{\prime}$, which is such that the composition $A^{\prime} \stackrel{\iota \otimes 1}{\longrightarrow} A \otimes A^{\prime} \stackrel{\sigma}{\longrightarrow} A^{\prime}$ is the identity map of $A^{\prime}$. Since the map $1 \otimes \iota \otimes 1: K_{s} \otimes A^{\prime} \longrightarrow K_{s} \otimes A \otimes A^{\prime}$ factors through the $k G$-module $P \otimes A^{\prime}$, we conclude that the identity map of $K_{s} \otimes A^{\prime}$ factors through $P \otimes A^{\prime}$ as well. Since $A^{\prime}$ is $k$-projective, the $k G$-module $P \otimes A^{\prime}$ is projective; hence, being a direct summand of it, the $k G$-module $K_{s} \otimes A^{\prime}$ is projective as well. Then, the exact sequence

$$
0 \longrightarrow K_{s} \otimes A^{\prime} \longrightarrow P_{s-1} \otimes A^{\prime} \longrightarrow \cdots \longrightarrow P_{0} \otimes A^{\prime} \longrightarrow k \otimes A^{\prime} \longrightarrow 0
$$

provides us with a projective resolution of the $k G$-module $k \otimes A^{\prime}=A^{\prime}$ of length $s$, and hence $\operatorname{pd}_{k G} A^{\prime} \leq s<\infty$.

(iv) $\rightarrow$ (vi): The assumptions made on $k$ imply that we have an equality spli $k G=$ silp $k G$ (cf. [13, Theorem 4.4]); therefore, the result will follow if we show that any injective $k G$-module has finite projective dimension. To that end, let $I$ be an injective $k G$-module. Then, the $k$-split monomorphism of $k G$-modules $\iota: k \longrightarrow A$ induces a monomorphism of $k G$-modules $1 \otimes \iota: I \longrightarrow I \otimes A$, which is split in view of the injectivity of $I$. Hence, we are reduced to showing that the $k G$-module $I \otimes A$ has finite projective dimension. Let $P_{*} \longrightarrow I \longrightarrow 0$ be a projective resolution of $I$ and assume that gl.dim $k=n$. Then, in the exact sequence

$$
0 \longrightarrow \Omega^{n} I \longrightarrow P_{n-1} \longrightarrow \cdots \longrightarrow P_{0} \longrightarrow I \longrightarrow 0
$$

the $k G$-modules $\Omega^{n} I, P_{n-1}, \ldots, P_{0}$ are $k$-projective. Since $A$ is $k$-projective (and hence $k$-flat), there is an induced exact sequence of $k G$-modules

$$
0 \longrightarrow \Omega^{n} I \otimes A \longrightarrow P_{n-1} \otimes A \longrightarrow \cdots \longrightarrow P_{0} \otimes A \longrightarrow I \otimes A \longrightarrow 0 .
$$

Hence, the finiteness of $\operatorname{pd}_{k G}(I \otimes A)$ will follow if we show that $\operatorname{pd}_{k G}(L \otimes A)<\infty$ for any $k$-projective $k G$-module $L$. But this is clear since for such a $k G$-module $L$ we have an inequality $\operatorname{pd}_{k G}(L \otimes A) \leq \operatorname{pd}_{k G} A<\infty 3$

(vi) $\rightarrow$ (i): Let $M$ be an arbitrary $k G$-module. Then, as shown by Gedrich and Gruenberg in [16, §4], the finiteness of spli $k G$ implies the existence of an acyclic complex $\left(P_{*}, \partial_{*}\right)$ of projective $k G$-modules which coincides with a projective resolution of $M$ in sufficiently large degrees. In view of the finiteness of silp $k G$, any projective $k G$-module has finite injective dimension; hence, the complex $\left(P_{*}, \partial_{*}\right)$ is totally acyclic, i.e. it is a strong complete projective resolution of $M$. The existence of such a resolution shows that the $k G$-module $M$ has finite Gorenstein projective dimension, as needed.

Remarks 6.5. (i) Let $k$ be a commutative ring, $G$ a group and $M$ a $k$-projective $k G$ module. We assume that there exists a $k$-split monomorphism of $k G$-modules $\iota$ : $k \longrightarrow A$, where $A$ is $k$-projective, such that the image of $1 \otimes \iota \in \operatorname{Hom}_{k G}(M, M \otimes A)$ vanishes in $\widehat{\operatorname{Ext}}_{k G}^{0}(M, M \otimes A)$. Then, the same arguments that were used in the proof of the implication $(\mathrm{v}) \rightarrow(\mathrm{iv})$ in Theorem 6.4 may be employed in order to show that there exists a $k$-split monomorphism of $k G$-modules $k \longrightarrow A^{\prime}$, where $A^{\prime}$ is $k$-projective, such that $\operatorname{pd}_{k G}\left(M \otimes A^{\prime}\right)<\infty$. This result is closely related to the finiteness criterion proved by Cornick and Kropholler in 12, Lemma 2.2 and Remark 2.4]. We avoid any assumption about the existence of a multiplicative structure on $A$, at the expense of replacing $A$ with $A^{\prime}$ (which is still a $k$-projective $k G$-module that contains $k$ as a $k$-split $k G$-submodule).

\footnotetext{
${ }^{3}$ The above argument shows that spli $k G \leq \operatorname{gl} \operatorname{dim} k+\operatorname{pd}_{k G} A$.
} 
(ii) In the case where $k$ is the ring of integers, the search for a module $A$ with the properties stated in Theorem 6.4(iv) was motivated by certain considerations in the study of free group actions on finite dimensional homotopy spheres (cf. 23], [24]).

\section{ACKNOWLEDGEMENTS}

It is a pleasure to thank A. Beligiannis and the anonymous referee for their very helpful comments and suggestions that improved the paper.

\section{REFERENCES}

[1] Anneaux de Gorenstein, et torsion en algèbre commutative (French), Séminaire d'Algèbre Commutative dirigé par Pierre Samuel, 1966/67. Texte rédigé, d'après des exposés de Maurice Auslander, Marquerite Mangeney, Christian Peskine et Lucien Szpiro. École Normale Supérieure de Jeunes Filles, Secrétariat mathématique, Paris, 1967. MR0225844 (37 \#1435)

[2] Maurice Auslander and Mark Bridger, Stable module theory, Memoirs of the American Mathematical Society, No. 94, American Mathematical Society, Providence, R.I., 1969. MR0269685 (42 \#4580)

[3] Maurice Auslander and Ragnar-Olaf Buchweitz, The homological theory of maximal CohenMacaulay approximations (English, with French summary), Mém. Soc. Math. France (N.S.) 38 (1989), 5-37. Colloque en l'honneur de Pierre Samuel (Orsay, 1987). MR.1044344 (91h:13010)

[4] Luchezar L. Avramov and Alex Martsinkovsky, Absolute, relative, and Tate cohomology of modules of finite Gorenstein dimension, Proc. London Math. Soc. (3) 85 (2002), no. 2, 393440, DOI 10.1112/S0024611502013527. MR1912056 (2003g:16009)

[5] Luchezar L. Avramov and Oana Veliche, Stable cohomology over local rings, Adv. Math. 213 (2007), no. 1, 93-139, DOI 10.1016/j.aim.2006.11.012. MR2331239 (2008f:13020)

[6] Abdolnaser Bahlekeh, Fotini Dembegioti, and Olympia Talelli, Gorenstein dimension and proper actions, Bull. Lond. Math. Soc. 41 (2009), no. 5, 859-871, DOI 10.1112/blms/bdp063. MR2557467 (2010k:20084)

[7] D. J. Benson and Jon F. Carlson, Products in negative cohomology, J. Pure Appl. Algebra 82 (1992), no. 2, 107-129, DOI 10.1016/0022-4049(92)90116-W. MR.1182934 (93i:20058)

[8] Kenneth S. Brown, Cohomology of groups, Graduate Texts in Mathematics, vol. 87, SpringerVerlag, New York, 1982. MR672956 (83k:20002)

[9] Henri Cartan and Samuel Eilenberg, Homological algebra, Princeton University Press, Princeton, N.J., 1956. MR0077480 (17,1040e)

[10] Lars Winther Christensen, Gorenstein dimensions, Lecture Notes in Mathematics, vol. 1747, Springer-Verlag, Berlin, 2000. MR.1799866 (2002e:13032)

[11] Lars Winther Christensen, Anders Frankild, and Henrik Holm, On Gorenstein projective, injective and flat dimensions - a functorial description with applications, J. Algebra 302 (2006), no. 1, 231-279, DOI 10.1016/j.jalgebra.2005.12.007. MR2236602 (2007h:13022)

[12] Jonathan Cornick and Peter H. Kropholler, Homological finiteness conditions for modules over group algebras, J. London Math. Soc. (2) 58 (1998), no. 1, 49-62, DOI 10.1112/S0024610798005729. MR1666074 (99k:20105)

[13] Ioannis Emmanouil, On certain cohomological invariants of groups, Adv. Math. 225 (2010), no. 6, 3446-3462, DOI 10.1016/j.aim.2010.06.007. MR2729012(2011m:20122)

[14] Edgar E. Enochs and Overtoun M. G. Jenda, Gorenstein injective and projective modules, Math. Z. 220 (1995), no. 4, 611-633, DOI 10.1007/BF02572634. MR1363858 (97c:16011)

[15] F. Thomas Farrell, An extension of Tate cohomology to a class of infinite groups, J. Pure Appl. Algebra 10 (1977/78), no. 2, 153-161. MR0470103 (57 \#9870)

[16] T. V. Gedrich and K. W. Gruenberg, Complete cohomological functors on groups, Topology Appl. 25 (1987), no. 2, 203-223, DOI 10.1016/0166-8641(87)90015-0. Singapore topology conference (Singapore, 1985). MR.884544 (89h:20073)

[17] François Goichot, Homologie de Tate-Vogel équivariante (French, with English summary), J. Pure Appl. Algebra 82 (1992), no. 1, 39-64, DOI 10.1016/0022-4049(92)90009-5. MR1181092 (94d:55014) 
[18] Henrik Holm, Gorenstein homological dimensions, J. Pure Appl. Algebra 189 (2004), no. 1-3, 167-193, DOI 10.1016/j.jpaa.2003.11.007. MR2038564 (2004k:16013)

[19] Henrik Holm, Rings with finite Gorenstein injective dimension, Proc. Amer. Math. Soc. 132 (2004), no. 5, 1279-1283 (electronic), DOI 10.1090/S0002-9939-03-07466-5. MR2053331 (2005a:13031)

[20] Peter H. Kropholler, On groups of type (FP) $)_{\infty}$, J. Pure Appl. Algebra 90 (1993), no. 1, 55-67, DOI 10.1016/0022-4049(93)90136-H. MR1246274 (94j:20051b)

[21] Guido Mislin, Tate cohomology for arbitrary groups via satellites, Topology Appl. 56 (1994), no. 3, 293-300, DOI 10.1016/0166-8641(94)90081-7. MR.1269317(95c:20072)

[22] Brita E. A. Nucinkis, Complete cohomology for arbitrary rings using injectives, J. Pure Appl. Algebra 131 (1998), no. 3, 297-318, DOI 10.1016/S0022-4049(97)00082-0. MR1637023 (99h:16012)

[23] Olympia Talelli, Periodicity in group cohomology and complete resolutions, Bull. London Math. Soc. 37 (2005), no. 4, 547-554, DOI 10.1112/S0024609305004273. MR2143734 (2006d:20095)

[24] C. T. C. Wall, Finiteness conditions for CW-complexes, Ann. of Math. (2) 81 (1965), 56-69. MR0171284(30 \#1515)

Department of Mathematics, University of Athens, Athens 15784, Greece

E-mail address: emmanoui@math.uoa.gr

Department of Mathematics, University of Athens, Athens 15784, Greece

E-mail address: otalelli@math.uoa.gr 